\title{
Low-intensity ultrasound enhances the chemosensitivity of hepatocellular carcinoma cells to cisplatin via altering the miR-34a/c-Met axis
}

\author{
PANPAN LI, JUANJUAN ZHANG, FUCHUN LI, YANYAN YU and YINGHONG CHEN \\ Department of Ultrasonography, Huaihe Hospital of Henan University, Kaifeng, Henan 475000, P.R. China
}

Received November 10, 2018; Accepted May 17, 2019

DOI: $10.3892 /$ ijmm.2019.4205

\begin{abstract}
Recently, the use of low-intensity ultrasound (LIUS) combined with chemotherapeutic agents is widely used in clinical practice, mainly for the treatment of cancer; however, the mechanisms as to how LIUS enhances the antitumor effects of these agents are not fully understood. The aim of the present study was to explore the synergistic antitumor effects and mechanisms of cisplatin (DDP) combined with LIUS (LIUS-DDP) in hepatocellular carcinoma (HCC). We reported that LIUS effectively enhanced Huh7 and HCCLM3 cell sensitivity to a low concentration of DDP. Reverse transcription-quantitative polymerase chain reaction analysis revealed that LIUS could increase the expression of microRNA-34a (miR-34a) in HCC cells following DDP treatment. In addition, LIUS-DDP significantly increased intracellular reactive oxygen species (ROS) levels in vitro, and the upregulation of miR-34a induced by LIUS-DDP was reversed by the ROS scavenger $\mathrm{N}$-acetylcysteine, suggesting that LIUS upregulates the expression of miR-34a via production of ROS. In addition, knockdown of miR-34a in HCC cells significantly suppressed the synergistic effects of LIUS-DDP treatment. Conversely, overexpression of miR-34a enhanced these synergistic effects. The results of a dual-luciferase assay indicated that c-Met, a well-known oncogene, was a target of miR-34a. We also determined that LIUS-DDP treatment inhibited the expression of c-Met, possibly due to increased ROS production, which upregulated miR-34a expression. Furthermore, overexpression of c-Met reversed the synergistic effects of LIUS-DDP treatment. Our findings suggest that LIUS could enhance the chemosensitivity of HCC cells to DDP by altering the miR-34a/c-Met axis. Therefore, DDP combined with LIUS may be a potential therapeutic application for the clinical treatment of patients with HCC.
\end{abstract}

Correspondence to: Professor Yinghong Chen, Department of Ultrasonography, Huaihe Hospital of Henan University, 8 Baobei Road, Kaifeng, Henan 475000, P.R. China

E-mail: yinghongchenyh@163.com

Key words: low-intensity ultrasound, cisplatin, hepatocellular carcinoma, microRNA-34a/c-Met axis

\section{Introduction}

Hepatocellular carcinoma (HCC) is one of the most prevalent malignant human tumors $(1,2)$. As of aggressive metastasis, recurrence and drug resistance, the overall 5-year survival rate of patients with HCC remains unsatisfactory and is <20\% (3). Cisplatin (DDP) is one of the most frequently used anticancer drugs and the front line option for the treatment of HCC; however, the clinical applications of DDP are limited largely due to severe side effects and chemoresistance (4).

Therapeutic ultrasound (US), particularly the use of low-intensity ultrasound (LIUS), has gained increasing attention in recent years as numerous studies have reported the synergistic effects of chemotherapeutic drugs and LIUS in cancer therapy. For example, Fan et al (5) revealed that effective low dosages of doxorubicin in combination with LIUS can inhibit cell proliferation, migration and invasion in oral squamous cell carcinoma. In a murine lymphoma, Tomizawa et al (6) reported that the combination of intraperitoneal bleomycin and US suppressed tumor growth. Emerging evidence demonstrated that excessive reactive oxygen species (ROS) production may be the key mechanism of US-enhanced chemotherapy (5). Hu et al (7) showed that combined US and 5-fluorouracil treatment regulated the expression of apoptosis-associated proteins via ROS in HCC; however, the mechanisms as to how LIUS enhances the antitumor effects of these agents are not fully understood.

MicroRNAs (miRNAs/miRs) are a class of endogenous small noncoding RNAs that regulate gene expression at the post-transcription level (8). Previously, the dysregulation of miRNAs and ROS were observed in human cancers, and extensive research showed that ROS contributed to the initiation and progression of carcinogenesis via the regulation of miRNAs (9-11). For example, elevating ROS levels by ionizing radiation induced profound alterations in global miRNA expression profiles of normal human fibroblasts following $\mathrm{H}_{2} \mathrm{O}_{2}$ treatment (12). Jajoo et al (10) reported that high ROS levels contribute to the increased metastatic potential of the prostate cancer cells via the regulation of miRNA-21. Additionally, US was observed to increase ROS production to affect tumor cell damage and apoptosis (13). Taken together, we proposed that LIUS may affect the sensitivity of HCC to DDP by regulating the expression of miRNAs via the production of ROS.

In the present study, the synergistic antitumor effects of DDP combined with LIUS were investigated in HCC cells. We 
also explored the potential mechanism of LIUS combined with DDP that enhances the antitumor effect. This study aimed to provide novel insight into the use of LIUS in HCC therapy.

\section{Materials and methods}

Chemicals and antibodies. Cisplatin and $\mathrm{N}$-acetylcysteine (NAC) were obtained from Sigma-Aldrich (Merck KGaA). Mouse anti-c-Met (cat.no. sc-8057) and mouse anti- $\beta$-actin (cat. no. sc-47778) were obtained from Santa Cruz Biotechnology, Inc.

Cell culture. HCC, Huh7, HCCLM3 and 293 cell lines were obtained from the American Type Culture Collection. All cells were cultured in Dulbecco's modified Eagle's medium (DMEM; Invitrogen; Thermo Fisher Scientific,Inc.) containing with 10\% fetal bovine serum (FBS; Sigma-Aldrich; Merck $\mathrm{KGaA})$, penicillin $(100 \mathrm{U} / \mathrm{ml})$ and streptomycin $(100 \mathrm{mg} / \mathrm{ml})$ in an incubator with a humidified atmosphere and $5 \% \mathrm{CO}_{2}$ at $37^{\circ} \mathrm{C}$. Cells were treated with $\operatorname{DDP}(0,1,2,4,6$, and $8 \mu \mathrm{g} / \mathrm{ml})$ for $48 \mathrm{~h}$ at $37^{\circ} \mathrm{C}$; untreated cells served as a control. In some experiments, the ROS scavenger NAC $(10 \mathrm{mM})$ was added into cells at $1 \mathrm{~h}$ prior to the administration of cisplatin at $37^{\circ} \mathrm{C}$, immediately followed by US exposure.

LIUS. HCC cells were stimulated with rectangular pulse ultrasound generated by the system SonoPore KTAC-4000 (Nepa Gene, Co., Ltd.). For the sonication of cell culture, we used the following US parameters: Frequency $1.1 \mathrm{MHz}$, provided in a pulse wave mode with duty cycle $10 \%$ and a repetition frequency of $100 \mathrm{~Hz}$; the $\mathrm{Hz}$ intensity was $1.0 \mathrm{~W} / \mathrm{cm}^{2}$ and the duration of sonication was $10 \mathrm{sec}$. The protocol was conducted as previously described by $\mathrm{Hu}$ et al (7).

Cell viability and the half-maximal inhibitory concentration $\left(I C_{50}\right)$. Huh7 and HCCLM3 cells transfected with miRNAs and plasmids (described below) were seeded in 96-well plates at a density of 5,000 cells/well. Following cellular adhesion, DDP was added to the cultured cells at a concentration of $1 \mu \mathrm{g} / \mathrm{ml}$ and incubated for $48 \mathrm{~h}$ at $37^{\circ} \mathrm{C}$, followed by exposure to LIUS. At $6 \mathrm{~h}$ following LIUS treatment, cell viability was monitored using an MTT assay. Untreated cells served as a blank group. Cells treated with DDP treatment alone served as the control. MTT solution $(15 \mu \mathrm{l})$ was added to each well, and the cells were incubated at $37^{\circ} \mathrm{C}$ for another $4 \mathrm{~h}$. The optical density was measured at $490 \mathrm{~nm}$ using a microplate reader (ELX50; BioTek Instruments, Inc.). The $\mathrm{IC}_{50}$ was calculated according to the cell viability curve.

Cell apoptosis detection by flow cytometry. An Annexin V fluorescein isothiocyanate (FITC)-propidium iodide (PI) Apoptosis kit (Invitrogen; Thermo Fisher Scientific, Inc.) was used for apoptosis analysis. The treated and control cells were collected, and washed twice with PBS at $4^{\circ} \mathrm{C}$. Then, the cells were re-suspended in $490 \mu \mathrm{l}$ binding buffer containing $5 \mu \mathrm{l}$ Annexin V (Bio-Science, Co. Ltd.) and $5 \mu \mathrm{l}$ PI. After incubation at $4^{\circ} \mathrm{C}$ in the dark for $30 \mathrm{~min}$, the cells were sorted using a FACS Aria III (BD Biosciences) and analyzed with BD FACSDiva (version 6.2; BD Biosciences) software. Cells were stained simultaneously with Annexin V-FITC (green fluorescence) and the non-vital dye PI (red fluorescence) allowed the determination of viable cells (FITC-PI'), and early apoptotic $\left(\mathrm{FITC}^{+} \mathrm{PI}\right)$ and late apoptotic $\left(\mathrm{FITC}^{+} \mathrm{PI}^{+}\right)$, or necrotic cells $\left(\mathrm{FITC}^{-} \mathrm{PI}^{+}\right)$. The rate of apoptotic cells $\left(\mathrm{FITC}^{+} \mathrm{PI}\right.$ and FITC $^{+} \mathrm{PI}^{+}$) was then calculated according to a previous report (14).

Determination of ROS. ROS levels were assessed by using the oxidation sensitive probe 2',7'-dichlorofluorescin diacetate (DCFH-DA). The treated and control cells were incubated with $10 \mu \mathrm{M}$ DCFH-DA at $37^{\circ} \mathrm{C}$ for $30 \mathrm{~min}$, and then the cells were sorted using a FACS Aria III (BD Biosciences) at an excitation wavelength of $488 \mathrm{~nm}$ and an emission wavelength of $530 \mathrm{~nm}$ and analyzed with BD FACSDiva software.

Reverse transcription-quantitative polymerase chain reaction $(R T-q P C R)$. Total RNA was extracted from the treated and control cells using the miRNeasy mini kit (Qiagen, Inc.) according to the manufacturer's instructions. Total RNA was converted to cDNA and added with a universal tag by using a miScript II RT kit (Qiagen, Inc.) for $60 \mathrm{~min}$ at $37^{\circ} \mathrm{C}$. qPCR was performed using the Power SYBR-Green PCR master mix on an ABI 7900HT system (both Applied Biosystems; Thermo Fisher Scientific, Inc.). The expression of miRNAs and mRNA were normalized to that of U6 and GAPDH, respectively. Thermocycling conditions were as following: $95^{\circ} \mathrm{C}$ for $5 \mathrm{~min}$, followed by 40 cycles of $95^{\circ} \mathrm{C}$ for $30 \mathrm{sec}$ and $55^{\circ} \mathrm{C}$ for $25 \mathrm{sec}$, and extension at $72^{\circ} \mathrm{C}$ for $10 \mathrm{sec}$. The primer sequences were synthesized by the Sangon Biotech Co. Ltd; the sequences were as follows: miR-34a forward, 5'-GCGGCCAATCAGCAAGTA TACT-3' and reverse, 5'-GTGCAGGGTCCGAGGT-3'; miR-133b forward, 5'-GTCCCCTTCAACCAGCTACA-3' and reverse, 5'-GAGTGCAAAGGCACAGAACA-3'; miR-340 forward, 5'-ACACTCCAGCTGGGTTATAAAGCAATG AGA-3' and reverse, 5'-TGGTGTCGTGGAGTCG-3'; miR-130a forward, 5'-CTTGCCCCTAAAGAGGGGGA-3' and reverse, 5'-CGAGTCAAAGGCTCCCCA-3'; miR-199a-5p forward, 5'-ACACTCCAGCTGGGCCCAGTGTTCAGAC TAC-3' and reverse, 5'-CTCAACTGGTGTCGTGGAGTCGG CAA-3'; miR-125b forward, 5'-UCCCUGAGACCCUAACUU GUGA-3' and reverse, 5'-ACAAGUUAGGGUCUCAGGG AUU-3'; U6 forward, 5'-AAAGACCTGTACGCCAACAC-3' and reverse, 5'-GTCATACTCCTGCTTGCTGAT-3'; c-Met forward, 5'-CATCTCAGAACGGTTCATGCC-3' and reverse, 5'-TGCACAATCAGGCTACTGGG-3'; GAPDH forward, 5'-AGGTCGGTGTGAACGGATTTG-3' and reverse, 5'-TGTAGACCATGTAGTTGAGGTCA-3'.

Cell transfection. Huh7 and HCCLM3 cells $\left(1.0 \times 10^{5}\right.$ per well) were seeded and grown overnight in six-well plates into 6-well plates $(2,000,000$ cells/well) in DMEM supplemented with $10 \%$ FBS for $24 \mathrm{~h}$ at $37^{\circ} \mathrm{C}$. miR-34a mimics, miR-34a inhibitor and the corresponding negative controls (NCs) were purchased from Shanghai GenePharma Co., Ltd. In addition, the coding domain sequences of c-Met mRNA were amplified by PCR, and inserted into pcDNA 3.0 vector to enhance its expression (Invitrogen; Thermo Fisher Scientific, Inc.), named as pcDNA-c-Met. pcDNA 3.0 empty vector was used as the control. A total of $100 \mathrm{nM}$ miR-34a mimics, $100 \mathrm{nM}$ miR-34a inhibitor or $2 \mu \mathrm{g}$ pcDNA-c-Met were transfected into Huh7 
and HCCLM3 cells using Lipofectamine ${ }^{\circledR} 2000$ (Invitrogen; Thermo Fisher Scientific, Inc.) following manufacturer's instructions. After $48 \mathrm{~h}$ transfection, cells were harvested for subsequent analysis.

Target gene analyses of miR-34a. Bioinformatics tools, including TargetScan 7.0 (targetscan.org/) and miRanda (microrna.org/), were used to predict the potential target genes of miR-34a.

Luciferase reporter assay. The 3'-untranslated region (UTR) of c-Met with wild-type (WT) or mutant (Mut) binding sites for miR-34a, was amplified and cloned into the pGL3 vector (Promega Corporation) to generate pGL3-WT-c-Met-3'-UTR or pGL3-Mut-c-Met-3'-UTR, respectively. For the luciferase reporter assay, cells were co-transfected with the luciferase reporter vectors and miR-34a mimics, miR-34a inhibitor or the corresponding NCs. The pRL-TK plasmid (Promega Corporation) was used as a normalizing control. After $48 \mathrm{~h}$ of incubation, luciferase activity was analyzed using the Dual-Luciferase Reporter Assay System (Promega Corporation).

Western blot analysis. Total protein was extracted using radioimmunoprecipitation assay lysis buffer (cat. no. P0013K; Beyotime Institute of Biotechnology) and the protein concentration was measured using a Bicinchoninic Acid assay kit (Pierce; Thermo Fisher Scientific, Inc.). Total protein (50 $\mu \mathrm{g})$ was loaded and separated by $15 \%$ SDS-PAGE and transferred to polyvinylidene difluoride membranes (GE Healthcare) by electroblotting. Primary antibodies against c-Met $(1: 1,000)$ and $\beta$-actin $(1: 3,000)$ were applied to the membrane at $4^{\circ} \mathrm{C}$ overnight. After incubating with goat anti-mouse IgG-horseradish peroxidase antibody (cat. no. sc2005; 1:10,000; Santa Cruz Biotechnology, Inc.) for $1 \mathrm{~h}$ at room temperature, the bands were detected using an enhanced chemiluminescence kit (GE Healthcare). The intensity of the bands was analyzed by ImageJ software (version 1.46; National Institutes of Health).

Statistical analysis. Statistical analyses were performed with SPSS 13.0 software (SPSS, Inc.). Data were expressed as the mean \pm standard deviation of three independent experiments. One-way analysis of variance followed by a Tukey's post-hoc test was applied to compare differences between multiple groups. $\mathrm{P}<0.05$ was considered to indicate a statistically significant difference.

\section{Results}

LIUS enhances the anticancer activity of cisplatin in vitro. Extensive investigations have demonstrated that the use of LIUS in combination with certain chemotherapeutic agents may be an important novel therapeutic strategy in treating human cancers (15-17). Thus, we sought to investigate whether LIUS enhanced the anticancer activity of DDP in vitro.

The present study determined the effects of DDP on the growth of HCC cells, Huh7 cells treated with DDP of different concentrations for $48 \mathrm{~h}$, by an MTT Assay. As shown in Fig. 1A and B, the viability of Huh7 and HCCLM3 cells was inhibited in a dose-dependent manner. DDP at $1 \mu \mathrm{g} / \mathrm{ml}$ was recommended for the following analysis as a slight anticancer effect was observed. As shown in Fig. $1 \mathrm{C}$, the $\mathrm{IC}_{50}$ values of Huh7 cells with or without LIUS treatment were $5.362 \pm 0.080$ and $3.922 \pm 0.095 \mathrm{ng} / \mathrm{ml}$, respectively, and the differences were statistically significant. Similarly, the $\mathrm{IC}_{50}$ values of HCCLM3 cells with or without LIUS treatment were $3.781 \pm 0.053$ and $2.179 \pm 0.262 \mathrm{ng} / \mathrm{ml}$, respectively; significant differences were observed between cells treated with or without LIUS. These results indicate that LIUS could effectively increase the sensitivity of HCC cells to DDP. To determine whether the reduction in cell viability was associated with cell apoptosis, flow cytometry was applied to detect the apoptotic rate of Huh7 and HCCLM3 cells following LIUS + DDP treatment. In the DDP group, DDP increased apoptosis by $9.5 \pm 2.4 \%$, in the LIUS group, apoptosis increased by $8.4 \pm 1.3 \%$, but in the LIUS + DDP group, the apoptotic rate was significantly increased by $35.5 \pm 2.3 \%$ in Huh7 cells compared with the control and DDP groups (Fig. 1D). In addition, the apoptotic rate in the LIUS + DDP group was significantly increased by $41.6 \pm 2.1 \%$ compared with the control and DDP groups; in the DDP and LIUS groups, apoptosis was increased by $7.8 \pm 2.5$ and $10.6 \pm 1.5 \%$, respectively in HCCLM3 cells (Fig. 1E). These data suggest that LIUS may improve the antitumor effects of DDP by increasing DDP sensitivity and DDP-induced apoptosis.

LIUS enhances intracellular ROS levels and miR-34a expression. It is widely accepted that the biological effects elicited by LIUS are predominantly due to the generation of ROS $(18,19)$. Providing that ROS can alter the expression of certain miRNAs $(20,21)$, we investigated whether the synergistic antitumor effects of LIUS occurs by altering the expression of miRNAs. Thus, we selected six miRNAs that have been reported to be regulated by ROS for further validation, including miR-34a (22,23), miR-133b (24), miR-340 (25), miR-130a (26), miR-199a-5p (27) and miR-125b (28). The results of RT-qPCR showed that LIUS significantly increased the expression of miR-34a in DDP-treated Huh7 and HCCLM3 cells compared with the control; however, no significant changes in expression were observed for miR-133b, miR-340, miR-130a, miR-199a-5p and miR-125b (Fig. 2A-F). In addition, the accumulation of ROS was detected. The results demonstrated that compared with the control group, the fluorescence intensity of ROS significantly increased in the DDP group compared with untreated cells; however, a stronger increase was observed in the LIUS + DDP group (Fig. 2G). Of note, the upregulation of miR-34a expression induced by LIUS was reversed in Huh7 and HCCLM3 cells that were pre-treated with the ROS scavenger NAC, suggesting that ROS modulated the expression of miR-34a (Fig. 2H). These findings indicated that the effects of LIUS may be associated with the induction of miR-34a mediated by ROS.

miR-34a expression is mediated the synergistic antitumor effects of LIUS and DDP. We then examined whether miR-34a participates in the synergistic antitumor effects of LIUS with DDP. The miRNA transfection efficiency of miR-34a in cultured Huh7 and HCCLM3 were determined; the results of RT-qPCR showed that the expression of miR-34a was significantly increased/decreased after miR-34a mimics/inhibitor 
A

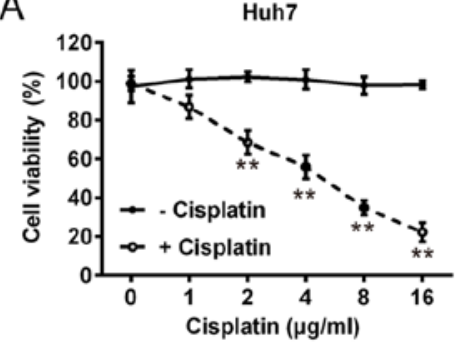

$\mathrm{B}$

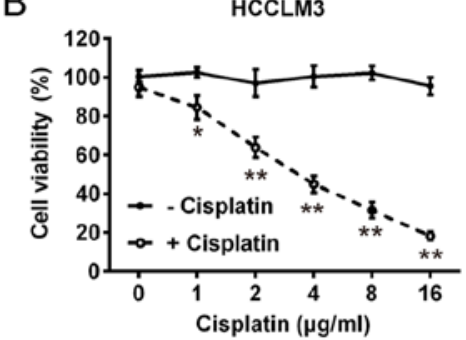

C

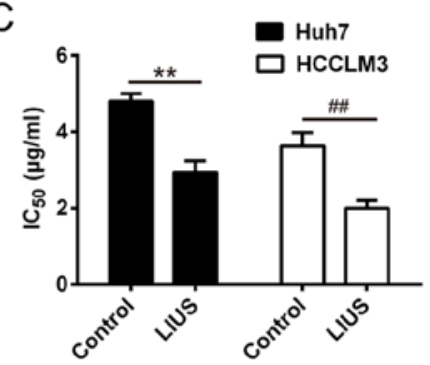

D

Huh7
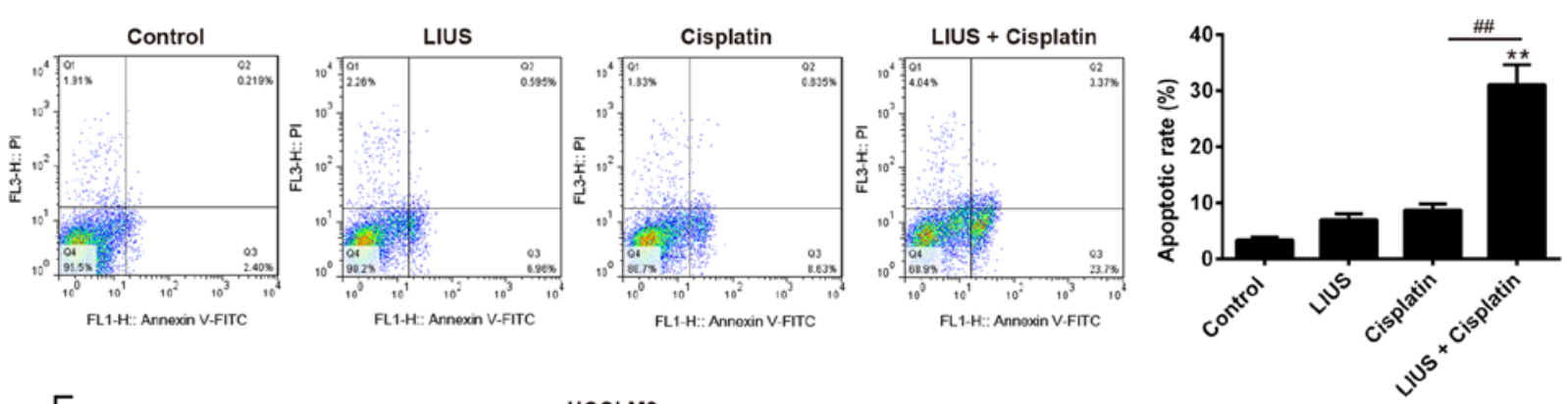

$\mathrm{E}$

HCCLM3
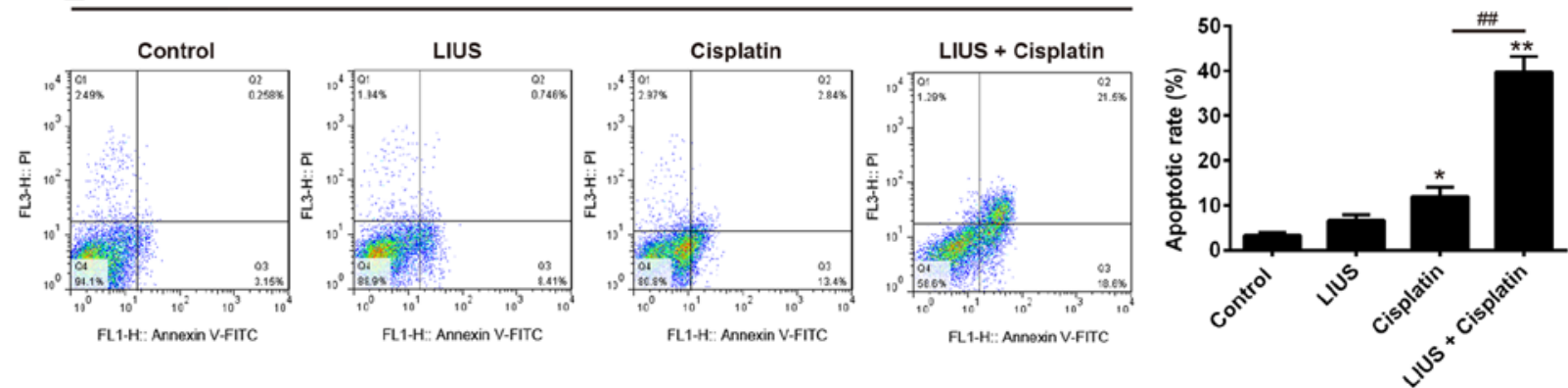

Figure 1. LIUS enhanced the antitumor activity of cisplatin in hepatocellular carcinoma cells. Huh7 and HCCLM3 cells were seeded in 96-well plates at a density of 5,000 cells/well. The cells were treated with different concentrations of cisplatin and/or LIUS for $48 \mathrm{~h}$, and the cells used for further analysis. (A and B) Cell viability was monitored using an MTT assay. (C) The $\mathrm{IC}_{50}$ was calculated according to the cell viability curve. (D and E) Cell apoptosis was detected by flow cytometry. Data are presented as the mean \pm standard deviation. $n=3,{ }^{*} \mathrm{P}<0.05,{ }^{* *} \mathrm{P}<0.01$ vs. Control group; ${ }^{\# \prime} \mathrm{P}<0.01$ vs. Cisplatin or Control group. LIUS, low-intensity ultrasound; $\mathrm{IC}_{50}$, half-maximal inhibitory concentration; FITC, fluorescein isothiocyanate.

transfection, respectively, compared with the corresponding NC (Fig. 3A and B). Subsequently, Huh7 and HCCLM3 cells were pre-treated with miR-34a inhibitor or inhibitor NC, followed by LIUS and DDP treatment. DDP treatment was used as control group. As anticipated, the expression of miR-34a was significantly increased in Huh7 and HCCLM3 cells compared to that in the control group; however, this promotive effect was inhibited when miR-34a was knocked down (Fig. 3C). In addition, cell viability and apoptosis were assessed by an MTT assay and flow cytometry, respectively. The results showed that LIUS + DDP treatment inhibited cell viability compared with the control group, whereas this inhibitory effect was reversed by miR-34a knock down (Fig. 3D). The increased apoptosis ratio induced by LIUS + DDP was also attenuated by miR-34a inhibitor (Fig. 3E). Additionally, Huh7 and HCCLM3 cells were transfected with miR-34a mimics, followed by LIUS and DDP treatment. We reported that miR-34a was overexpressed following miR-34a mimics transfection in Huh7 and HCCLM3 cells under LIUS + DDP treatment compared with the control (Fig. 3F). Furthermore, overexpression of miR-34a enhanced the synergistic antitumor effects of LIUS with DDP, as observed by a significant reduction in cell viability and increased apoptosis (Fig. 3G and H).
These results suggested that the synergistic antitumor effects of LIUS and DDP may be mediated by miR-34a.

c-Met is a direct target of miR-34a. To further investigate the mechanisms by which miR-34a mediated the synergistic antitumor effects of LIUS and DDP in HCC cells, we determined the potential target genes of miR-34a using the miRanda and TargetScan 6.1 databases. As presented in Fig. 4A, miR-34a contained a sequence complementary to c-Met. In addition, c-Met reported as a target of miR-34a in colorectal cancer cell and human mesothelial cells $(29,30)$. To further validate these findings, the c-Met-3'UTR wt luciferase reporter systems with the putative binding sites and c-Met-3'UTR mut reporter systems with the mutation sites were constructed in 293 cells. The results of the luciferase reporter assays showed that introduction of miR-34a mimics significantly inhibited, while miR-34a inhibitor increased the relative luciferase activity of constructs containing the c-Met-3'UTR compared with the respective $\mathrm{NC}$ group. However, the luciferase activity of the reporters containing the mutant binding site exhibited marked alterations (Fig. 4B). To further confirm that c-Met is negatively regulated by miR-34a, western blot analysis was conducted to determine the protein expression levels of c-Met. The results 
A

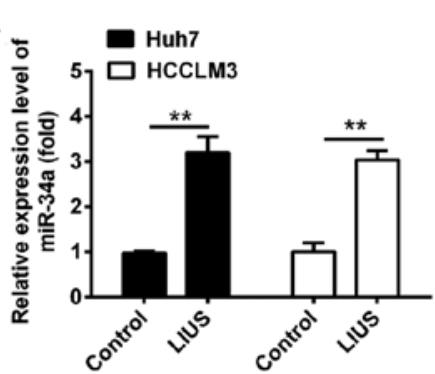

D

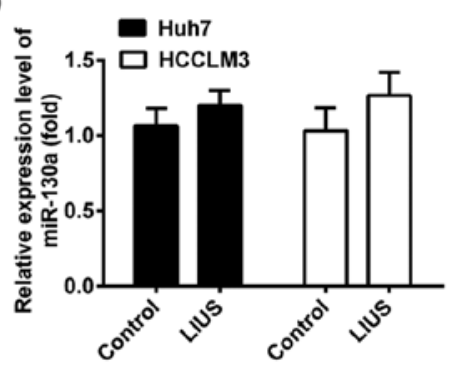

B

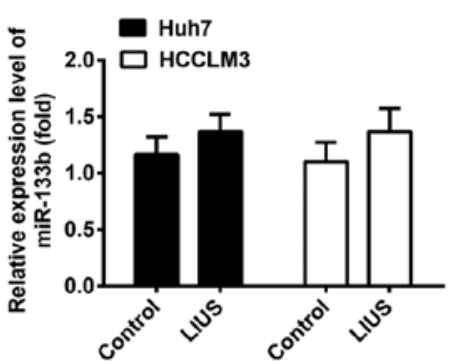

E

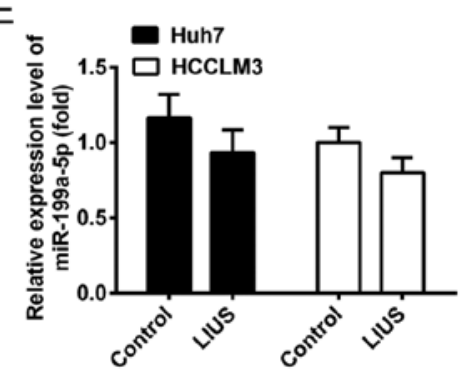

C

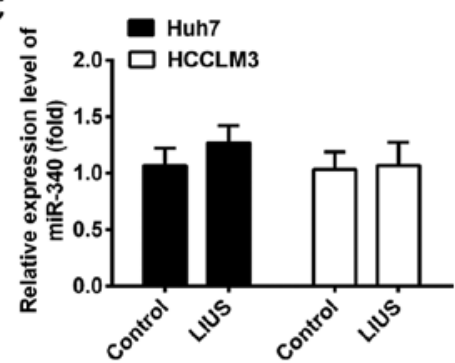

F

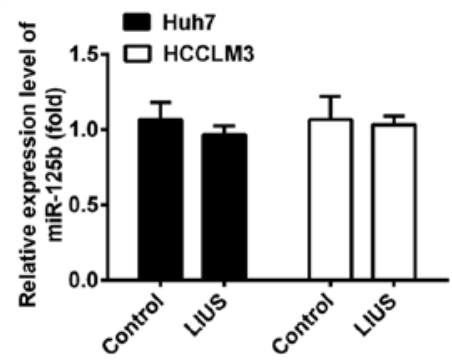

G

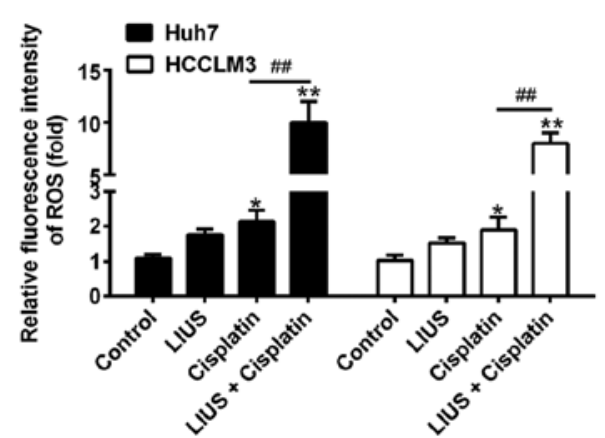

$\mathrm{H}$

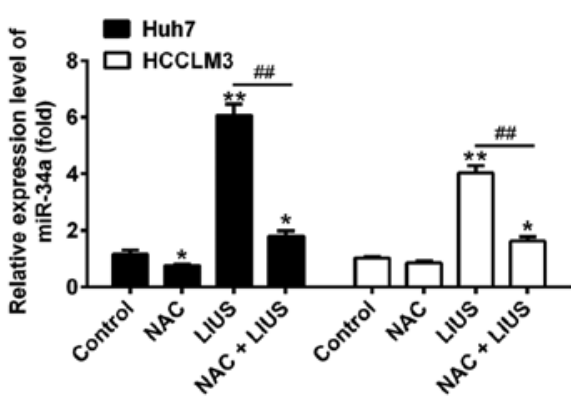

Figure 2. LIUS enhances the intracellular ROS levels and miR-34a expression. The cells were treated with cisplatin (1 $\mu \mathrm{g} / \mathrm{ml})$ and/or LIUS for $48 \mathrm{~h}$. (A-F) Expression levels of miR-34a, miR-133b, miR-340, miR-130a, miR-199a-5p and miR-125b were measured by RT-qPCR. (G) The relative levels of intracellular ROS were quantified using $2^{\prime}, 7^{\prime}$-dichlorofluorescin diacetate. $\mathrm{n}=3$. ${ }^{*} \mathrm{P}<0.05,{ }^{* *} \mathrm{P}<0.01$ vs. Control group; ${ }^{\# \prime} \mathrm{P}<0.01$ vs. Cisplatin. (H) Expression of miR-34a was measured by RT-qPCR after cisplatin and/or LIUS treatment in the presence or absence of the ROS scavenger NAC. Data are presented as the mean \pm standard deviation. $n=3,{ }^{*} \mathrm{P}<0.05,{ }^{* *} \mathrm{P}<0.01$ vs. Control group; ${ }^{\#} \mathrm{P}<0.01$ vs. LIUS group. LIUS, low-intensity ultrasound; miR, microRNA; NAC, $\mathrm{N}$-acetylcysteine; ROS, reactive oxygen species; RT-qPCR, reverse transcription-quantitative polymerase chain reaction.

revealed that c-Met was significantly downregulated following the overexpression of miR-34a, but was upregulated after knockdown in Huh7 and HCCLM3 cells compared with the corresponding NC group (Fig. 4C and D). Subsequently, the effects of LIUS and DDP on the expression of c-Met were detected in Huh7 and HCCLM3 cells by western blot analysis. As presented in Fig. 4E and F, DDP significantly reduced the expression of c-Met compared with untreated cells, whereas LIUS + DDP significantly inhibited expression in Huh7 and HCCLM3 cells. Conversely, c-Met expression was significantly increased following treatment with the ROS scavenger NAC. Our results suggested that the combined treatment of LIUS and DDP could upregulate the expression of miR-34a via ROS production and reduce the expression of c-Met.

Restoration of $c$-Met reverses the antitumor effects of LIUS combined with DDP. After confirming c-Met was a direct target of miR-34a in HCC cells, we further investigated whether the antitumor effects of LIUS combined with DDP is mediated via the downregulation of c-Met. Huh7 and HCCLM3 cells were transfected with pcDNA-c-Met plasmids, followed by LIUS and DDP treatment. Western blot analysis revealed the upregulation of c-Met by the pcDNA-c-Met plasmids, compared with that in the pcDNA-vector group (Fig. 5A). It was also observed that LIUS suppressed the endogenous expression of c-Met in DDP-treated Huh7 and HCCLM3 cells, which was reversed by pcDNA-c-Met (Fig. 5B). Then, cell viability and apoptosis were evaluated by an MTT assay and flow cytometry, respectively. As presented in Fig. 5C and D, the reduction in cell viability induced by LIUS + DDP was reversed by pcDNA-c-Met overexpression. Furthermore, flow cytometry indicated that the promotion of apoptosis induced by LIUS + DDP was attenuated when c-Met was overexpressed (Fig. 5E and F). These findings suggest that miR-34a may have mediated the synergistic antitumor effects of LIUS combined with DDP in HCC cells by targeting c-Met.

\section{Discussion}

In the present study, we first revealed that LIUS could enhance the antitumor activity of DDP in HCC cells. Based on in vitro explorations, it was proposed that miR-34a and the regulation 

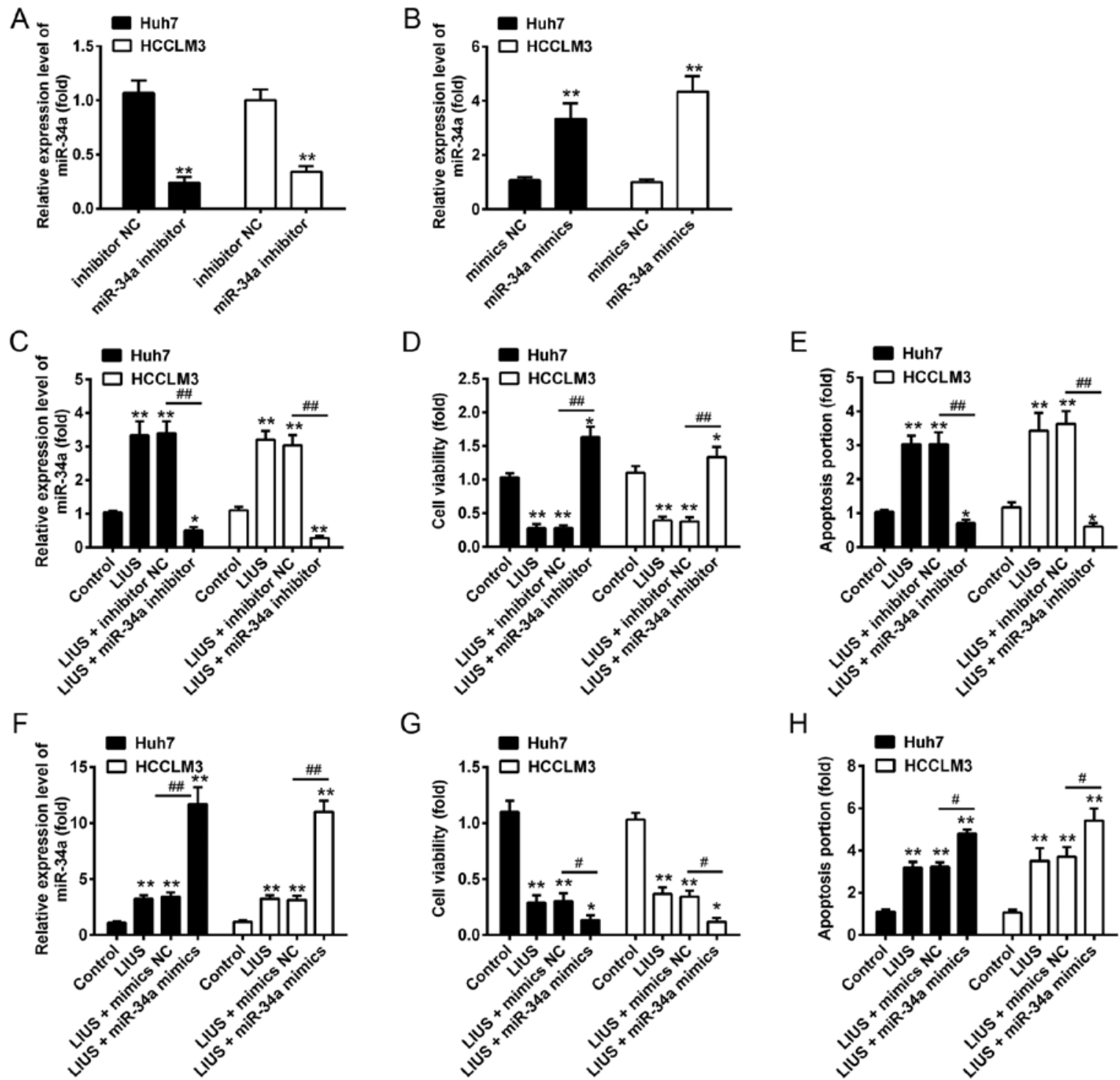

Figure 3. miR-34a mediates the synergistic antitumor effects of LIUS and cisplatin. Huh7 and HCCLM3 cells were seeded in 96-well plates at a density of 5,000 cells/well and transfected with miR-34a inhibitor and miR-34a mimics. The cells were treated with cisplatin and/or LIUS for $48 \mathrm{~h}$, and then cells were used for further analysis. (A and B) miRNA transfection efficiency of miR-34a in cultured Huh7 and HCCLM3 was assessed by RT-qPCR. (C and F) The expression of miR-34a was measured by RT-qPCR. (D and G) Cell viability was monitored using an MTT assay. (E and H) Cell apoptosis was detected by flow cytometry. Data are presented as the mean \pm standard deviation. $n=3,{ }^{*} \mathrm{P}<0.05,{ }^{* *} \mathrm{P}<0.01$ vs. Control group; ${ }^{*} \mathrm{P}<0.05,{ }^{\# \#} \mathrm{P}<0.01$ vs. LIUS + inhibitor NC or mimics NC. LIUS, low-intensity ultrasound; miR, microRNA; NC, negative control; RT-qPCR, reverse transcription-quantitative polymerase chain reaction.

of c-Met were key mechanisms involved in the synergistic antitumor effects of LIUS and DDP combination treatment.

In recent years, the role of LIUS in cancer therapy has been implicated in vitro and in vivo $(13,31,32)$. Few investigations demonstrated that LIUS enhanced the antitumor effects of several chemotherapeutic agents, including doxorubicin, cyclophosphamide, docetaxel and DDP (33-36). Yoshida et al (15) had demonstrated that LIUS could increase the uptake of doxorubicin, and caused a synergistic enhancement in cell killing and the induction of apoptosis in human lymphoma U937 cells. The synergistic efficacy of chemotherapy and US has also been studied in mouse tumor models. For example, Li et al (37) found the treatment of scutellarin and ultrasound significantly delayed human tongue carcinoma xenograft growth, inhibited tumor angiogenesis and lymphanigoenesis in tumor-bearing Balb/c mice. Although applications of LIUS are still in the process of investigation, LIUS has distinct potential as a technique for cancer treatment, particular in cases of DDP resistance (16). In the present study, it was revealed that LIUS effectively enhanced HCC cell sensitivity to a low concentration of DDP, indicating that LIUS could enhance the antitumor effects of DDP in HCC. However, the mechanisms underlying the synergistic effects of LIUS combined with DDP in HCC are yet to be elucidated.

Increasing experimental evidence has indicated that the cell damage induced by the synergistic effects of US and drugs may contribute to the generation ROS $(7,37)$. For example, LIUS combined with 5-aminolevulinic acid significantly suppressed the growth of human tongue squamous carcinoma in vitro and in vivo via the production of ROS (38). Huang et al (39) showed that new quinolone antibiotics could exhibit notable antitumor activity under US irradiation, and that generation of ROS is involved in this process. In our study, it was observed that LIUS stimulation increased DDP-mediated ROS generation in HCC cells, suggesting that LIUS enhanced the antitumor effects of DDP via 
A

Position 2165-2171 of c-Met 3' UTR

wt c-Met 3'-UTR: 5'...GAAUUAGAUACUUGUCACUGCCU... 3'

IIIIII

hsa-miR-34a-5p: 3' ...UGUUGGUCGAUUCUGUGACGGU... 5'

mut c-Met 3'-UTR:5' ...GAAUUAGAUACUUGUCGACCUAU... 3'

C

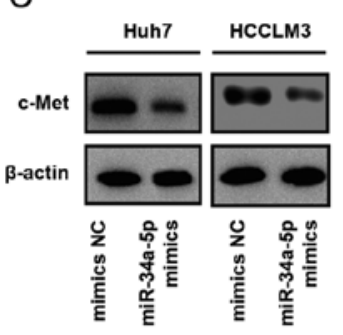

E

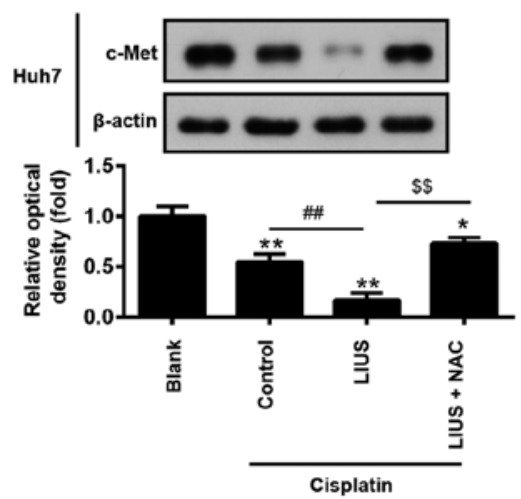

B

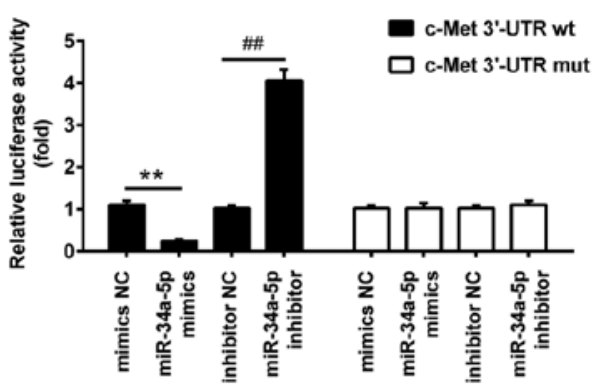

D
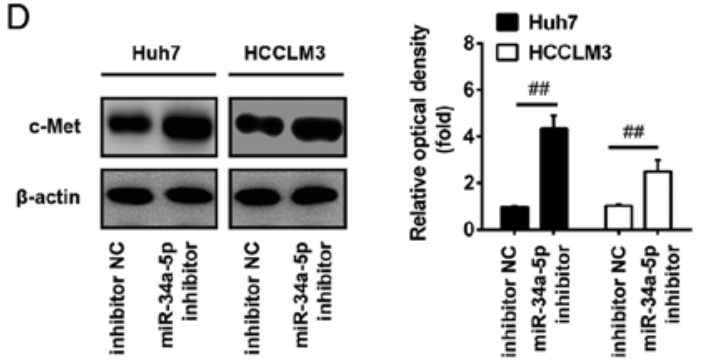

F

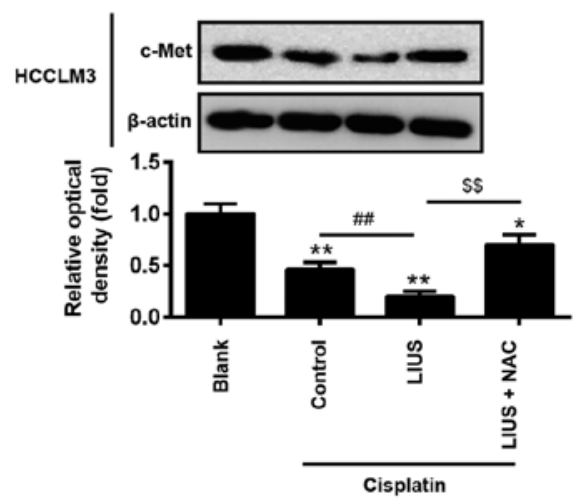

Figure 4. c-Met is a direct target of miR-34a. (A) The predicted binding sites for miR-34a on c-Met. (B) Luciferase activity in 293 cells co-transfected with miR-34a mimics, miR-34a inhibitor and luciferase reporters containing c-Met or MUT 3'-UTR. Histogram indicates the values of luciferase measured $48 \mathrm{~h}$ after transfection. Data are presented as the mean \pm standard deviation. $\mathrm{n}=3,{ }^{* *} \mathrm{P}<0.01$ vs. mimic $\mathrm{NC}$; ${ }^{\# \#} \mathrm{P}<0.01$ vs. inhibitor $\mathrm{NC}$ group. (C and $\left.\mathrm{D}\right) \mathrm{miR}-34 \mathrm{a}$ mimics, miR-34a inhibitor and controls were transfected into Huh7 and HCCLM3 cells, then after $48 \mathrm{~h}$ transfection, the protein expression levels of c-Met were detected by western blotting. Data are presented as the mean \pm standard deviation. $\mathrm{n}=3,{ }^{* *} \mathrm{P}<0.01 \mathrm{vs}$. mimics $\mathrm{NC}$ group; ${ }^{\# \#} \mathrm{P}<0.01 \mathrm{vs}$. inhibitor $\mathrm{NC}$ group. (E and F) The expression of c-Met was measured by western blotting after cisplatin and/or LIUS treatment in the presence or absence of the ROS scavenger NAC. Untreated cells served as Blank group. Cells treated with DDP treatment alone served as the control. Data are presented as the mean \pm standard deviation. $\mathrm{n}=3,{ }^{*} \mathrm{P}<0.05,{ }^{* *} \mathrm{P}<0.01$ vs. Blank group; ${ }^{\# \#} \mathrm{P}<0.01$ vs. Cisplatin group; ${ }^{\$} \mathrm{P}<0.01$ vs. Cisplatin + LIUS group. LIUS, low-intensity ultrasound; miR, microRNA; Mut, mutant; NAC, N-acetylcysteine; NC, negative control; Wt, wild type.

ROS generation. Recently, it was reported that ROS is involved in the initiation of cancer metastasis through the regulation of miRNA expression (11), including miR-21 in prostate cancer (10) and miR-125b in breast cancer (40). Therefore, we proposed that novel miRNAs regulated by ROS may serve an important role in the synergistic antitumor effects of LIUS combined with DDP. In this study, we selected six miRNAs regulated by ROS including miR-34a (22,23), miR-133b (24), miR-34a (25), miR-130a (26), miR-199a-5p (27) and miR-125b (28), and found that miR-34a was significantly increased following combination treatment. Additionally, the upregulation of miR-34a could be abrogated when ROS were scavenged, indicating that LIUS induced the expression of miR-34a via ROS production. Furthermore, several studies have reported that miR-34a functions as a tumor suppressor in a variety of human cancers, such as pancreatic cancer (41), colorectal cancer (42), breast cancer (43) and lung cancer (44). Subsequently, we investigated the function of miR-34a in the synergistic antitumor effects of LIUS combined with DDP. The results demonstrated that knockdown of miR-34a inhibited the synergistic antitumor effects of LIUS combined with DDP, whereas overexpression of miR-34a enhanced these synergistic antitumor effects. These results suggested that the synergistic antitumor effects of LIUS and DDP combination occurred via the ROS-mediated upregulation of miR-34a.

The mesenchymal-epithelial transition factor c-Met is a well-known oncogene, and its aberrant activation is responsible for the growth, progression, invasion and poor prognosis of various solid tumors, including HCC $(45,46)$. Importantly, overexpression of c-Met is frequently observed in patients with HCC, and is closely associated with the clinicopathological characteristics of HCC (47). Of note, targeting c-Met is emerging as an anticancer therapeutic option for HCC (48). Interestingly, c-Met has been reported to be a direct target of miR-34a in colorectal cancer and human mesothelial cells $(29,30)$. In the present study, c-Met was also identified as a target of miR-34a. Additionally, LIUS-DDP significantly 
A

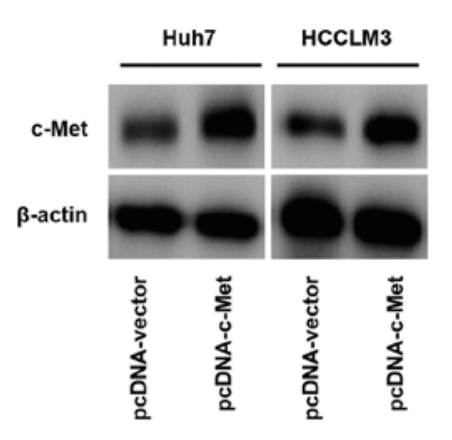

D

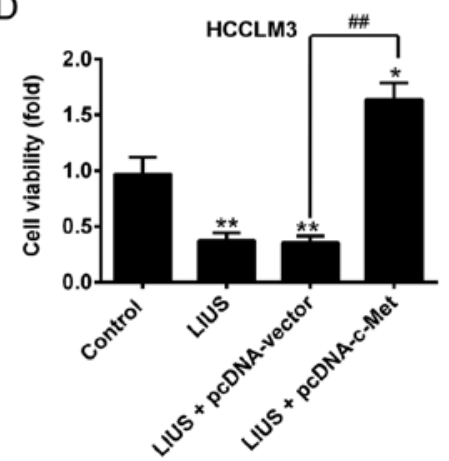

B

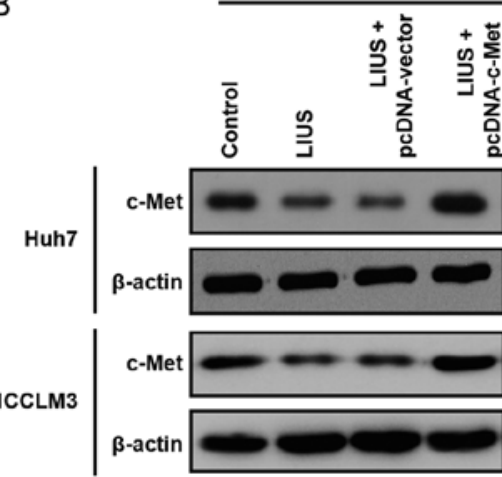

E

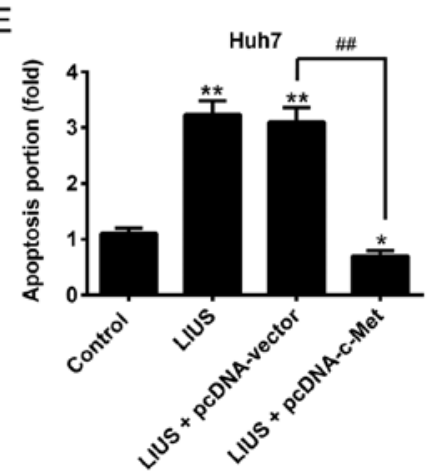

C

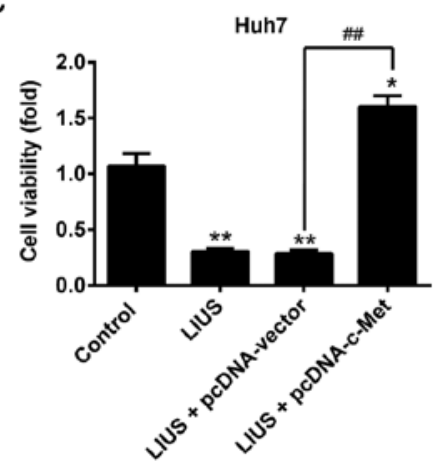

$\mathrm{F}$

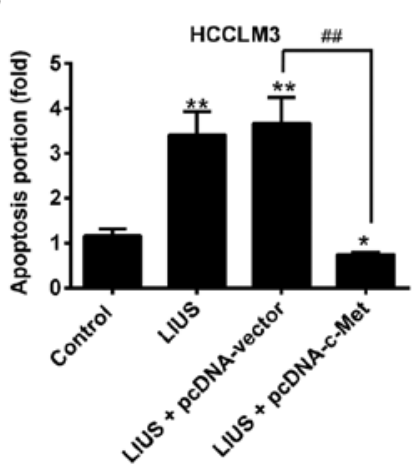

Figure 5. c-Met mediates the antitumor effects of LIUS combined with cisplatin. Huh7 and HCCLM3 cells were seeded in 96-well plates at a density of 5,000 cells/well and transfected with pcDNA-c-Met. The cells were treated with DDP and/or LIUS for $48 \mathrm{~h}$ and the cell viability and cell apoptosis was monitored. (A) The transfection efficiency of pcDNA-c-Met plasmids in cultured Huh7 and HCCLM3 was assessed by western blotting. (B) The protein expression levels of c-Met were detected by western blotting in cultured Huh7 and HCCLM3. (C and D) Cell viability was measured by an MTT assay. (E and F) Cell apoptosis was detected by flow cytometry. Data are presented as the mean \pm standard deviation. $\mathrm{n}=3,{ }^{*} \mathrm{P}<0.05,{ }^{* * *} \mathrm{P}<0.01 \mathrm{vs}$. Control group; ${ }^{\# \#} \mathrm{P}<0.01$ vs. LIUS + pcDNA-vector. LIUS, low-intensity ultrasound.

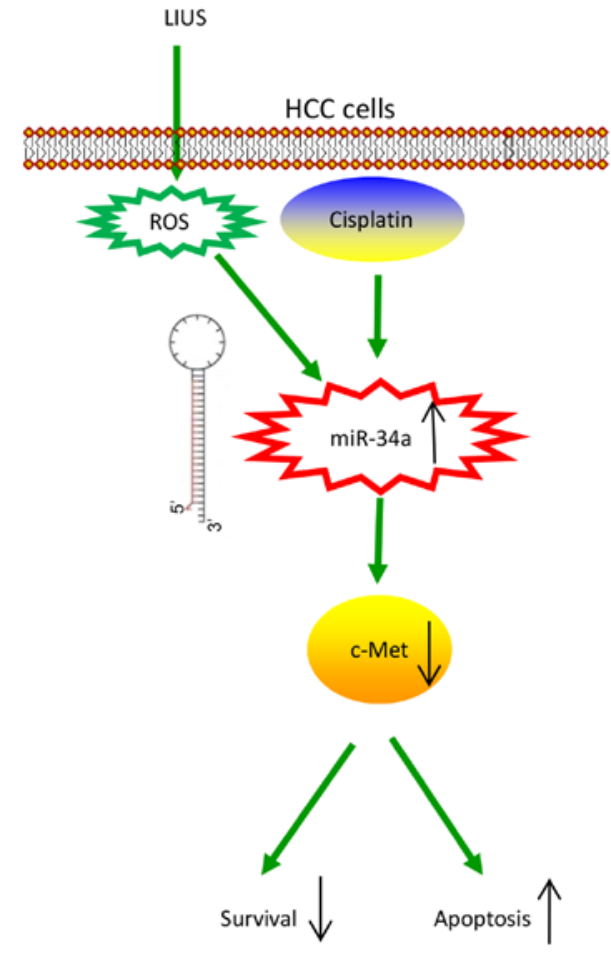

Figure 6. Schematic diagram showing that LIUS enhances the anticancer effects of cisplatin via increased ROS production. This induced the expression of miR-34a and reduced that of c-Met, a well-known oncogene, to suppress cell viability and induce cell apoptosis. LIUS, LIUS, low-intensity ultrasound; HCC, hepatocellular carcinoma; miR, microRNA; ROS, reactive oxygen species. decreased the expression of c-Met, but this inhibitory effect was reversed when ROS were scavenged. Furthermore, we observed that the synergistic antitumor effects of LIUS-DDP were abolished when c-Met was overexpressed. These results suggested that LIUS combined with DDP exerts the synergistic antitumor effects via downregulation of c-Met by the ROS-mediated upregulation of miR-34a.

In conclusion, we demonstrated that LIUS enhanced the anticancer effects of DDP by increasing ROS production, which induced the expression of miR-34a and reduced that of c-Met, a well-known oncogene, to suppress cell viability and induce apoptosis (Fig. 6). Our findings indicated that LIUS combined with DDP may be a promising novel therapy, particularly for drug-resistant patients with HCC.

\section{Acknowledgements}

Not applicable.

\section{Funding}

No funding was received.

\section{Availability of data and materials}

All data generated or analyzed during this study are included in this published article. 


\section{Authors' contributions}

PL, JZ, FL and YY performed the experiments, contributed to data analysis and wrote the paper. PL, JZ, FL and YY analyzed the data. YC made substantial contributions to the concept of the study, contributed to data analysis and acquired experimental materials. All authors read and approved the final manuscript.

\section{Ethics approval and consent to participate}

The present study was approved by the Huaihe Hospital of Henan University Ethics Committees.

\section{Patient consent for publication}

Not applicable.

\section{Competing interests}

The authors declare that they have no competing interests.

\section{References}

1. Sapisochin G, de Sevilla EF, Echeverri J and Charco R: Management of 'very early' hepatocellular carcinoma on cirrhotic patients. World J Hepatol 6: 766-775, 2014.

2. Faloppi L, Scartozzi M, Maccaroni E, Di Pietro Paolo M, Berardi R, Del Prete M and Cascinu S: Evolving strategies for the treatment of hepatocellular carcinoma: From clinical-guided to molecularly-tailored therapeutic options. Cancer Treat Rev 37 $169-177,2011$

3. Allemani C, Matsuda T, Di Carlo V, Harewood R, Matz M, Nikšić M, Bonaventure A, Valkov M, Johnson CJ, Estève J, et al; CONCORD Working Group: Global surveillance of trends in cancer survival 2000-14 (CONCORD-3): Analysis of individual records for 37513025 patients diagnosed with one of 18 cancers from 322 population-based registries in 71 countries. Lancet 391: $1023-1075,2018$

4. Thomas MB, O'Beirne JP, Furuse J, Chan AT, Abou-Alfa G and Johnson P: Systemic therapy for hepatocellular carcinoma: Cytotoxic chemotherapy, targeted therapy and immunotherapy. Ann Surg Oncol 15: 1008-1014, 2008.

5. Fan H, Li H, Liu G, Cong W, Zhao H, Cao W and Zheng J: Doxorubicin combined with low intensity ultrasound suppresses the growth of oral squamous cell carcinoma in culture and in xenografts. J Exp Clin Cancer Res 36: 163, 2017.

6. Tomizawa M, Ebara M, Saisho H, Sakiyama S and Tagawa M: Irradiation with ultrasound of low output intensity increased chemosensitivity of subcutaneous solid tumors to an anti-cancer agent. Cancer Lett 173: 31-35, 2001.

7. Hu Z, Lv G, Li Y, Li E, Li H, Zhou Q, Yang B and Cao W: Enhancement of anti-tumor effects of 5-fluorouracil on hepatocellular carcinoma by low-intensity ultrasound. J Exp Clin Cancer Res 35: 71, 2016.

8. Bartel DP: MicroRNAs: Genomics, biogenesis, mechanism, and function. Cell 116: 281-297, 2004.

9. Melnik BC: miR-21: An environmental driver of malignant melanoma? J Transl Med 13: 202, 2015.

10. Jajoo S, Mukherjea D, Kaur T, Sheehan KE, Sheth S, Borse V, Rybak LP and Ramkumar V: Essential role of NADPH oxidase-dependent reactive oxygen species generation in regulating microRNA-21 expression and function in prostate cancer. Antioxid Redox Signal 19: 1863-1876, 2013.

11. He J and Jiang BH: Interplay between Reactive oxygen species and microRNAs in cancer. Curr Pharmacol Rep 2: 82-90, 2016.

12. Simone NL, Soule BP, Ly D, Saleh AD, Savage JE, Degraff W, Cook J, Harris CC, Gius D and Mitchell JB: Ionizing radiation-induced oxidative stress alters miRNA expression. PLoS One 4: e6377, 2009.

13. Yu T, Wang Z and Mason TJ: A review of research into the uses of low level ultrasound in cancer therapy. Ultrason Sonochem 11: 95-103, 2004.
14. Qi XF, Kim DH, Yoon YS, Kim SK, Cai DQ, Teng YC, Shim KY and Lee KJ: Involvement of oxidative stress in simvastatin-induced apoptosis of murine CT26 colon carcinoma cells. Toxicol Lett 199: 277-287, 2010.

15. Yoshida T, Kondo T, Ogawa R, Feril LB Jr, Zhao QL, Watanabe A and Tsukada K: Combination of doxorubicin and low-intensity ultrasound causes a synergistic enhancement in cell killing and an additive enhancement in apoptosis induction in human lymphoma U937 cells. Cancer Chemother Pharmacol 61: $559-567,2008$.

16. Watanabe Y, Aoi A, Horie S, Tomita N, Mori S, Morikawa H, Matsumura Y, Vassaux G and Kodama T: Low-intensity ultrasound and microbubbles enhance the antitumor effect of cisplatin. Cancer Sci 99: 2525-2531, 2008

17. Masui T, Ota I, Kanno M, Yane K and Hosoi H: Low-intensity ultrasound enhances the anticancer activity of cetuximab in human head and neck cancer cells. Exp Ther Med 5: 11-16, 2013.

18. McHale AP, Callan JF, Nomikou N, Fowley C and Callan B: Sonodynamic therapy: Concept, mechanism and application to cancer treatment. Adv Exp Med Biol 880: 429-450, 2016.

19. Wan GY, Liu Y, Chen BW, Liu YY, Wang YS and Zhang N: Recent advances of sonodynamic therapy in cancer treatment. Cancer Biol Med 13: 325-338, 2016.

20. Lin J, Chuang CC and Zuo L: Potential roles of microRNAs and ROS in colorectal cancer: Diagnostic biomarkers and therapeutic targets. Oncotarget 8: 17328-17346, 2017.

21. Dando I, Cordani M, Dalla Pozza E, Biondani G, Donadelli M and Palmieri M: Antioxidant mechanisms and ROS-related microRNAs in cancer stem cells. Oxid Med Cell Longev 2015: 425708, 2015.

22. Li QC, Xu H, Wang X, Wang T and Wu J: miR-34a increases cisplatin sensitivity of osteosarcoma cells in vitro through up-regulation of c-Myc and Bim signal. Cancer Biomark 21: 135-144, 2017.

23. Song C, Lu P, Sun G, Yang L, Wang Z and Wang Z: miR-34a sensitizes lung cancer cells to cisplatin via $553 / \mathrm{miR}-34 \mathrm{a} / \mathrm{MYCN}$ axis. Biochem Biophys Res Commun 482: 22-27, 2017.

24. Zhuang Q, Zhou T, He C, Zhang S, Qiu Y, Luo B, Zhao R, Liu H, Lin Y and Lin Z: Protein phosphatase 2A-B558 enhances chemotherapy sensitivity of human hepatocellular carcinoma under the regulation of microRNA-133b. J Exp Clin Cancer Res 35: 67, 2016.

25. Shi L, Chen ZG, Wu LL, Zheng JJ, Yang JR, Chen XF, Chen ZQ, Liu CL, Chi SY, Zheng JY, et al: miR-340 reverses cisplatin resistance of hepatocellular carcinoma cell lines by targeting Nrf2-dependent antioxidant pathway. Asian Pac J Cancer Prev 15: 10439-10444, 2014.

26. Xu N, Shen C, Luo Y, Xia L, Xue F, Xia Q and Zhang J: Upregulated miR-130a increases drug resistance by regulating RUNX3 and Wnt signaling in cisplatin-treated HCC cell. Biochem Biophys Res Commun 425: 468-472, 2012.

27. Li Y, Jiang W, Hu Y, Da Z, Zeng C, Tu M, Deng Z and Xiao W: MicroRNA-199a-5p inhibits cisplatin-induced drug resistance via inhibition of autophagy in osteosarcoma cells. Oncol Lett 12: 4203-4208, 2016.

28. Zhang X, Yao J, Guo K, Huang H, Huai S, Ye R, Niu B, Ji T, Han $\mathrm{W}$ and Li J: The functional mechanism of miR-125b in gastric cancer and its effect on the chemosensitivity of cisplatin. Oncotarget 9: 2105-2119, 2017.

29. Luo Y, Ouyang J, Zhou D, Zhong S, Wen M, Ou W, Yu H, Jia L and Huang Y: Long noncoding RNA GAPLINC promotes cells migration and invasion in colorectal cancer cell by regulating miR-34a/c-MET signal pathway. Dig Dis Sci 63: 890-899, 2018.

30. Tanaka N, Toyooka S, Soh J, Tsukuda K, Shien K, Furukawa M, Muraoka T, Maki Y, Ueno T, Yamamoto H, et al: Downregulation of microRNA-34 induces cell proliferation and invasion of human mesothelial cells. Oncol Rep 29: 2169-2174, 2013.

31. Misík V and Riesz P: Free radical intermediates in sonodynamic therapy. Ann N Y Acad Sci 899: 335-348, 2000.

32. Rosenthal I, Sostaric JZ and Riesz P: Sonodynamic therapy-a review of the synergistic effects of drugs and ultrasound. Ultrason Sonochem 11: 349-363, 2004.

33. Tinkov S, Coester C, Serba S, Geis NA, Katus HA, Winter G and Bekeredjian R: New doxorubicin-loaded phospholipid microbubbles for targeted tumor therapy: in-vivo characterization. J Control Release 148: 368-372, 2010.

34. Todorova M, Agache V, Mortazavi O, Chen B, Karshafian R, Hynynen K, Man S, Kerbel RS and Goertz DE: Antitumor effects of combining metronomic chemotherapy with the antivascular action of ultrasound stimulated microbubbles. Int J Cancer 132: 2956-2966, 2013. 
35. Goertz DE, Todorova M, Mortazavi O, Agache V, Chen B, Karshafian R and Hynynen K: Antitumor effects of combining docetaxel (taxotere) with the antivascular action of ultrasound stimulated microbubbles. PLoS One 7: e52307, 2012.

36. Yu T, Yang Y, Liu S and Yu H: Ultrasound increases DNA damage attributable to cisplatin in cisplatin-resistant human ovarian cancer cells. Ultrasound Obstet Gynecol 33: 355-359, 2009.

37. Li H, Fan H, Wang Z, Zheng $\mathbf{J}$ and Cao W: Potentiation of scutellarin on human tongue carcinoma xenograft by low-intensity ultrasound. PLoS One 8: e59473, 2013.

38. Lv Y, Fang M, Zheng J, Yang B, Li H, Xiuzigao Z, Song W, Chen Y, Cao W: Low-intensity ultrasound combined with 5-aminolevulinic acid administration in the treatment of human tongue squamous carcinoma. Cell Physiol Biochem 30: 321-333, 2012.

39. Huang D, Okada K, Komori C, Itoi E and Suzuki T: Enhanced antitumor activity of ultrasonic irradiation in the presence of new quinolone antibiotics in vitro. Cancer Sci 95: 845-849, 2004.

40. Hu G, Zhao X, Wang J, Lv L, Wang C, Feng L, Shen L and Ren W: miR-125b regulates the drug-resistance of breast cancer cells to doxorubicin by targeting HAX-1. Oncol Lett 15: 1621-1629, 2018

41. Zhang QA, Xu HY, Chen D, et al: miR-34 increases in vitro PANC-1 cell sensitivity to gemcitabine via targeting Slug/PUMA. Cancer Biomark 21: 755-762, 2018.

42. Toyota M, Suzuki H, Sasaki Y, Maruyama R, Imai K, Shinomura Y and Tokino T: Epigenetic silencing of microRNA-34b/c and B-cell translocation gene 4 is associated with $\mathrm{CpG}$ island methylation in colorectal cancer. Cancer Res 68: 4123-4132, 2008.

43. Achari C, Winslow S, Ceder Y and Larsson C: Expression of miR-34c induces G2/M cell cycle arrest in breast cancer cells. BMC Cancer 14: 538, 2014.
44. Garofalo M, Jeon YJ, Nuovo GJ, Middleton J, Secchiero P, Joshi P, Alder H, Nazaryan N, Di Leva G, Romano G, et al: MiR-34a/c-dependent PDGFR- $\alpha / \beta$ downregulation inhibits tumorigenesis and enhances TRAIL-induced apoptosis in lung cancer. PLoS One 8: e67581, 2013.

45. Gherardi E, Birchmeier W, Birchmeier C and Vande Woude G: Targeting MET in cancer: Rationale and progress. Nat Rev Cancer 12: 89-103, 2012.

46. Awad MM, Oxnard GR, Jackman DM, Savukoski DO, Hall D, Shivdasani P, Heng JC, Dahlberg SE, Jänne PA, Verma S, et al: MET exon 14 mutations in non-small-cell lung cancer are associated with advanced age and stage-dependent MET genomic amplification and c-met overexpression. J Clin Oncol 34: 721-730, 2016.

47. Han P, Li H, Jiang X, Zhai B, Tan G, Zhao D, Qiao H, Liu B, Jiang $H$ and Sun X: Dual inhibition of Akt and c-Met as a second-line therapy following acquired resistance to sorafenib in hepatocellular carcinoma cells. Mol Oncol 11: 320-334, 2017.

48. de Rosamel L and Blanc JF: Emerging tyrosine kinase inhibitors for the treatment of hepatocellular carcinoma. Expert Opin Emerg Drugs 22: 175-190, 2017.

This work is licensed under a Creative Commons Attribution-NonCommercial-NoDerivatives 4.0 International (CC BY-NC-ND 4.0) License. 